\title{
Diffusion tensor magnetic resonance imaging of the optic nerves in pediatric hydrocephalus
}

\author{
*Katie Shpanskaya, BS, ${ }^{1}$ Jennifer L. Quon, MD, ${ }^{2}$ Robert M. Lober, MD, PhD, ${ }^{3}$ Sid Nair, ${ }^{4}$ \\ Eli Johnson, BS, ${ }^{1}$ Samuel H. Cheshier, MD, PhD, ${ }^{5}$ Michael S. B. Edwards, MD, ${ }^{6}$ \\ Gerald A. Grant, MD, ${ }^{6}$ and Kristen W. Yeom, MD ${ }^{4}$
}

\begin{abstract}
1Stanford University School of Medicine, Stanford; 2Department of Neurosurgery, Stanford University School of Medicine, Stanford, California; ${ }^{3}$ Department of Neurosurgery, Wright State University Boonshoft School of Medicine, Dayton, Ohio; ${ }^{4}$ Division of Pediatric Neuroradiology, Department of Radiology, Lucile Packard Children's Hospital, Stanford University School of Medicine, Stanford, California; ${ }^{5}$ Division of Pediatric Neurosurgery, Department of Neurosurgery, University of Utah, Salt Lake City, Utah; and ${ }^{6}$ Division of Pediatric Neurosurgery, Department of Neurosurgery, Lucile Packard Children's Hospital, Stanford University School of Medicine, Stanford, California
\end{abstract}

OBJECTIVE While conventional imaging can readily identify ventricular enlargement in hydrocephalus, structural changes that underlie microscopic tissue injury might be more difficult to capture. MRI-based diffusion tensor imaging (DTI) uses properties of water motion to uncover changes in the tissue microenvironment. The authors hypothesized that DTI can identify alterations in optic nerve microstructure in children with hydrocephalus.

METHODS The authors retrospectively reviewed 21 children (< 18 years old) who underwent DTI before and after neurosurgical intervention for acute obstructive hydrocephalus from posterior fossa tumors. Their optic nerve quantitative DTI metrics of mean diffusivity (MD) and fractional anisotropy (FA) were compared to those of 21 age-matched healthy controls.

RESULTS Patients with hydrocephalus had increased MD and decreased FA in bilateral optic nerves, compared to controls $(p<0.001)$. Normalization of bilateral optic nerve MD and FA on short-term follow-up (median 1 day) after neurosurgical intervention was observed, as was near-complete recovery of MD on long-term follow-up (median 1.8 years).

CONCLUSIONS DTI was used to demonstrate reversible alterations of optic nerve microstructure in children presenting acutely with obstructive hydrocephalus. Alterations in optic nerve MD and FA returned to near-normal levels on shortand long-term follow-up, suggesting that surgical intervention can restore optic nerve tissue microstructure. This technique is a safe, noninvasive imaging tool that quantifies alterations of neural tissue, with a potential role for evaluation of pediatric hydrocephalus.

https://thejns.org/doi/abs/10.3171/2019.9.FOCUS19619

KEYWORDS hydrocephalus; MRI; diffusion tensor imaging; fractional anisotropy; mean diffusivity; optic nerve

$\mathrm{H}$ YDROCEPHALUS is the most common neurosurgical condition treated by pediatric neurosurgeons and accounts for more than $\$ 2$ billion in health expenditures in the US alone. ${ }^{14}$ It is a complex condition characterized by the imbalance of CSF production and absorption, and can cause symptoms related to intracranial hypertension such as headaches, nausea, vomiting, and visual changes. ${ }^{1}$ Increased intracranial pressure (ICP) can also manifest as papilledema, or optic disc edema. How- ever, papilledema is a less sensitive indicator of increased ICP in children younger than 8 years. ${ }^{18}$ Several theories have been proposed regarding the pathogenesis of papilledema, one of which suggests that axoplasmic flow stasis on the nerve surface and in the prelaminar region of the nerve head causes swelling and disc edema. ${ }^{8}$ Accumulation of extracellular fluid around the nerve further contributes to increases in pressure. ${ }^{8}$ Animal studies suggest that optic disc edema may be observed as early as several hours after

ABBREVIATIONS DTI = diffusion tensor imaging; EVD = external ventricular drain; FA = fractional anisotropy; GRAPPA = generalized autocalibrating partially parallel acquisition; ICP = intracranial pressure; $\mathrm{MD}=$ mean diffusivity; OCT = optical coherence tomography; $\mathrm{PF}=$ posterior fossa; $\mathrm{ROI}=$ region of interest; $\mathrm{SNR}=$ signal-to-noise ratio.

SUBMITTED August 1, 2019. ACCEPTED September 4, 2019.

INCLUDE WHEN CITING DOI: 10.3171/2019.9.FOCUS19619.

${ }^{*}$ K.S. and J.L.Q. contributed equally to this work and share first authorship. 
a rise in ICP, with the diagnosis becoming more definitive at 1 week. ${ }^{8}$ Because the optic nerve sheath is anatomically continuous with the intracranial dura, optic nerve sheath fenestration may be beneficial in patients with optic disc edema and visual deterioration. ${ }^{8}$ Although papilledema usually resolves after relief of elevated ICP, in severe cases or with delayed treatment, more permanent visual deficits and chronic papilledema can occur.

While conventional neuroimaging studies such as CT or MRI can easily depict enlarged ventricular spaces, they do not provide quantitative information on the degree of tissue damage associated with hydrocephalus. Diffusion tensor imaging (DTI) is an advanced MRI technique that quantifies the diffusion properties of water molecules within brain tissue, specifically its magnitude and directionality. ${ }^{2}$ Thus, measurements of mean diffusivity (MD) and fractional anisotropy (FA) can be used to probe (in vivo) underlying microstructural changes of the cerebral white matter. ${ }^{11}$ High MD reflects cellular loss and a subsequent increase in extracellular water content. ${ }^{11}$ Increases in FA can indicate compressed white matter, whereas decreased FA occurs in the setting of tissue degradation. ${ }^{11}$

Previous studies have used DTI to examine white matter in adults with idiopathic hydrocephalus and found significant differences in MD and FA, ${ }^{7}$ which were relieved after normalization of ICP.,12 However, studies of pediatric hydrocephalus have largely focused on the corpus callosum, anterior and posterior limbs of the internal capsule, corticospinal tract, and superior and inferior longitudinal fasciculus. ${ }^{14}$ No studies have examined diffusion tensor-based microstructural changes of the optic nerve in children presenting with obstructive hydrocephalus. In this study, we hypothesized that diffusion tensor metrics are altered in the optic nerves of children presenting with acute hydrocephalus.

\section{Methods \\ Hydrocephalus Cohort}

We retrospectively reviewed all patients presenting with posterior fossa (PF) brain tumors at Lucile Packard Children's Hospital from January 2010 to January 2017, after IRB approval. The following inclusion criteria were used to identify the study cohort: patient obtained a baseline generalized autocalibrating partially parallel acquisition (GRAPPA)-DTI at 3T; presented with clinical signs of elevated ICP that required neurosurgical intervention (tumor resection and/or CSF diversion) within a week of the baseline DTI scan; radiographically confirmed ventriculomegaly; and received no prior medical/radiation therapy or permanent CSF diversion.

Chronic hydrocephalus may be difficult to diagnose and can be associated with chronic white matter injury or architectural changes. ${ }^{13}$ Thus, for this study purpose, we targeted patients who presented acutely with obstructive hydrocephalus requiring intervention. Specifically, we selected patients presenting with PF tumors, as this represented a more homogeneous group presenting with an obstructive source of hydrocephalus. We excluded patients with: chronic medical diseases; genetic disorders or syndromes (including neurofibromatosis type 1); cardiac disease; hemorrhage; vascular lesions (aneurysm, arteriovenous malformation, fistula, or stenoocclusive disease); prior strokes; concomitant supratentorial tumors; leptomeningeal seeding, or other tumors, including optic pathway lesions; or prior history of radiation or chemotherapy, given their potential impact on cerebral and optic nerve white matter. We also excluded patients with dental braces or other hardware that could corrupt the DTI data set.

\section{Controls}

Normal controls who had obtained GRAPPA-DTI at 3T as part of routine brain MRI were randomly selected from our database and matched for age and sedation status of the study subjects. This cohort has been described in more detail in previous studies. ${ }^{5,15}$ The control group consisted of children with no underlying neurological or ophthalmological diseases and a normal brain MRI, including the orbital contents. Examples of clinical reasons for obtaining MRI were isolated headaches, cholesteatoma of the middle ear, isolated facial lesions (e.g., hemangioma, glabella dermoid), scalp nevus, inflammatory nasal obstruction, short stature, and family history of aneurysms. Children with neurocutaneous syndromes, optic nerve atrophy or vision problems, ataxia, gaze abnormality, history of prematurity or cerebral palsy, or any chronic diseases (such as cancer) and previously treated neurological diseases, and prior or ongoing medical therapy were excluded from the control cohort.

\section{Clinical Assessment}

We reviewed the neurosurgical documentation regarding each patient's symptoms at presentation and after neurosurgical intervention, and recorded any visual signs or symptoms, including diplopia, blurry vision, visual loss, or papilledema.

\section{Imaging Methods}

GRAPPA-DTI was acquired at 3T MRI (Discovery 750, GE Medical Systems) with an 8-channel head coil and a twice-refocused generalized autocalibrating parallel acquisitions diffusion tensor echo planar imaging sequence (acquisition matrix $=128,{ }^{2}$ acceleration factor $=$ 3 , number of excitations $=3,25$ isotropically distributed diffusion directions with $b=1000 \mathrm{sec} / \mathrm{mm}^{2}$, five $b=0$ images, slice thickness/gap $=3 \mathrm{~mm} / 0 \mathrm{~mm}$, field of view $=20 \mathrm{~cm}$ ) using the reconstruction and motion-correction methods previously described..$^{17}$

\section{Imaging Analysis \\ Diffusion Tensor MRI}

FA and MD were obtained using the region of interest (ROI) analysis. A board-certified neuroradiology attending (K.W.Y.) blindly performed the ROI analysis (Fig. 1). Specifically, the reviewer selected the midsegment of the intraconal optic nerve with most optimal signal-to-noise ratio (SNR) on DTI. In cases in which the midportion of the optic nerve was incompletely imaged due to nerve tortuosity, volume averaging, or image slice selection, the reviewer selected a region slightly anterior or posterior to the midsegment that showed more robust SNR. The 

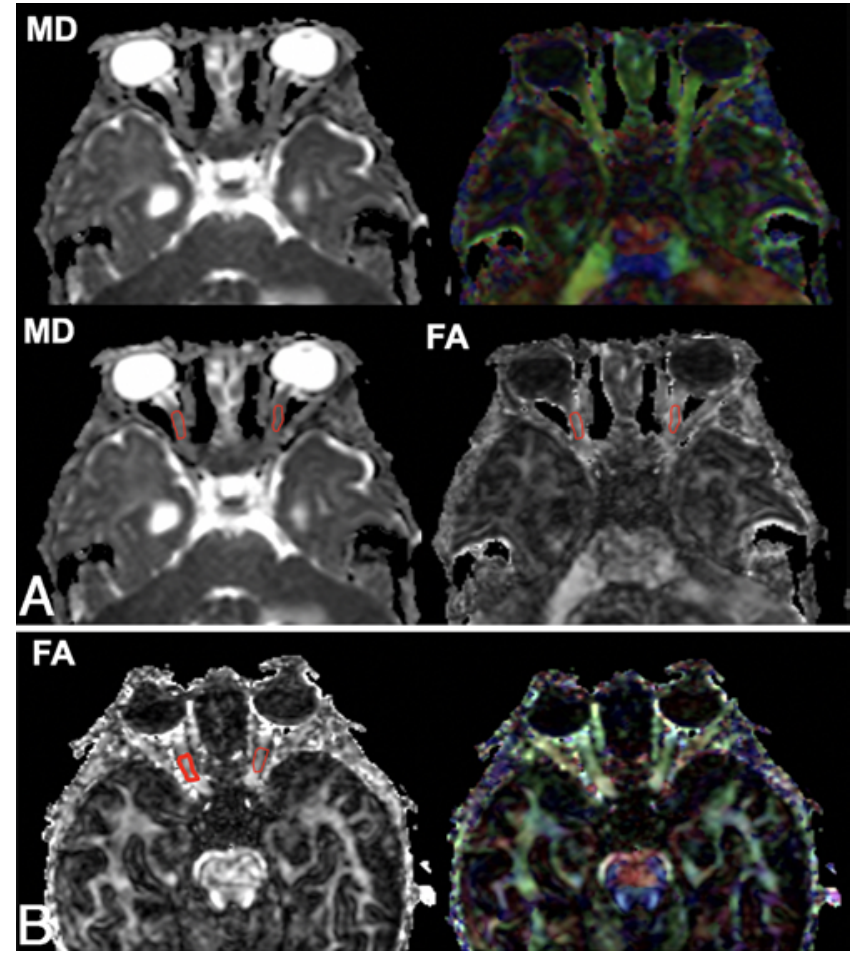

FIG. 1. Optic nerve ROI placement in a 21-month-old boy with hydrocephalus (A) and in a 14-month-old girl from the control group (B). ROls (red outlines) were placed along the midsegment of the bilateral optic nerves on both MD and FA maps. Example of before (upper) and after (lower) ROI placement is shown for MD (A). The color map was also utilized to help cross-reference optic nerves.

ROIs were adjusted based on the size of the optic nerve, but ranged in overall size from 10 to $15 \mathrm{~mm}^{2}$. A second blinded, board-certified neuroradiology attending (Dr. Michael Iv) independently assessed the data set for proper ROI placement.

DTI parameters for right and left optic nerves were averaged within each available time point. Averaged bilateral values were used in the subsequent analysis. The MRI data set was randomized, and the reviewers were blinded to clinical information, study group, status of neurosurgical intervention, and clinical outcome at all times. For comparison between diffusion metrics at baseline and after neurosurgical intervention, short-term follow-up (within 3 months since surgical intervention), and long-term follow-up (most recent follow-up at least 3 months after surgical intervention) GRAPPA-DTI studies performed at 3T were used. The reviewers were blinded to clinical information, study groups, status of neurosurgical intervention, and clinical outcome at all times.

\section{Other Imaging Features}

The bifrontal index was measured at the level of the caudate heads in each patient at baseline as a surrogate for overall ventricular size. We also recorded abnormal $\mathrm{T} 2$ hyperintense signal of the optic chiasm, optic chiasm bowing at presentation based on coronal $\mathrm{T} 2$ fast spin echo imaging of the orbits, and optic atrophy on the most recent
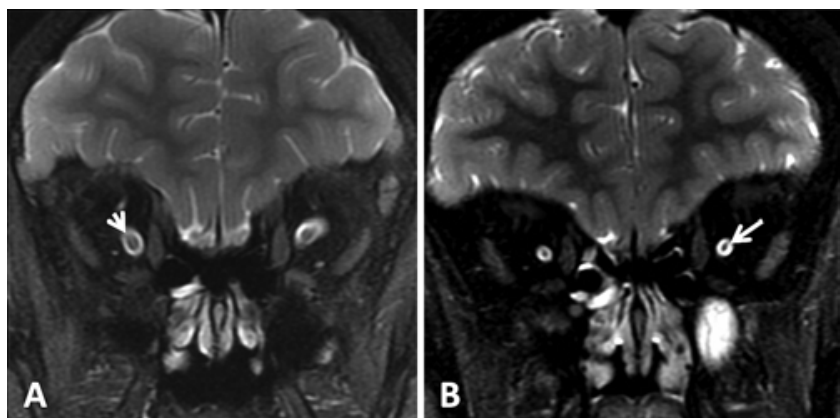

FIG. 2. A: Initial coronal T2-weighted MR image from a 6-year-old boy presenting with blindness and obstructive hydrocephalus from a large PF tumor. B: Seven-year follow-up T2 MR image showing optic nerve atrophy (arrow) compared to initial MRI (arrowhead in A).

follow-up MRI since neurosurgical intervention. Some of these features are shown in Fig. 2.

\section{Statistical Analysis}

Baseline comparison of optic nerve DTI parameters and global cerebral blood flow between patients with hydrocephalus and controls was analyzed using the nonparametric Mann-Whitney U-test for independent samples. Within-patient comparisons of DTI metrics before and after neurosurgical intervention at short- and long-term follow-up time points were performed using the Wilcoxon signed-rank test for paired samples, a nonparametric alternative to the paired-samples t-test. Pearson correlation analysis was conducted to assess the association of the degree of hydrocephalus (determined by ventricular size), patient age, and bilateral optic nerve DTI parameters in the preoperative hydrocephalus cohort. Differences in sex distribution between the control and hydrocephalus cohorts were investigated using the chi-square test. All statistical analyses were performed using the Statistical Package for Social Sciences (SPSS; version 25; IBM Corp.) with an a priori significance level of $\alpha=0.05$.

\section{Results}

\section{Demographics}

Of 33 patients presenting acutely with symptoms of hydrocephalus from PF tumors, 21 patients (median age 7.7 years, range 1.1-17 years) met the study criteria and were included. Patient demographics and clinical data are shown in Table 1 . The control group matched by age consisted of 21 children (15 boys), with an overall median age of 7.3 years (range 1.1-17 years). In the hydrocephalus group, the tumors included diffuse midline glioma $(\mathrm{n}=1)$, medulloblastoma $(n=13)$, pilocytic astrocytoma $(n=3)$, ependymoma $(\mathrm{n}=3)$, and atypical choroid plexus papilloma $(\mathrm{n}=1)$.

All 21 patients selected for this study presented with symptomatic obstructive hydrocephalus, 20 of whom underwent tumor resection. In 2 patients, CSF drainage was performed prior to MRI; in one, an external ventricular drain (EVD) was placed prior to MRI due to clinical instability, whereas in another, a lumbar puncture was per- 
TABLE 1. Obstructive hydrocephalus and control cohort characteristics

\begin{tabular}{|c|c|c|c|}
\hline Characteristic & Hydrocephalus & Control & $\mathrm{p}$ Value \\
\hline No. of patients & 21 & 21 & \\
\hline Age at initial MRI, yrs & & & 0.831 \\
\hline Median & 7.67 & 7.33 & \\
\hline Range & $1.1-17$ & $1.1-17$ & \\
\hline Sex & & & 0.513 \\
\hline Male & $13(61.9 \%)$ & $15(71.4 \%)$ & \\
\hline Female & $8(38.1 \%)$ & $6(28.6 \%)$ & \\
\hline \multicolumn{4}{|l|}{ Diagnosis } \\
\hline Medulloblastoma & 13 & & \\
\hline Pilocytic astrocytoma & 3 & & \\
\hline Ependymoma & 3 & & \\
\hline Choroid plexus papilloma & 1 & & \\
\hline Diffuse midline glioma & 1 & & \\
\hline \multicolumn{4}{|l|}{ Presenting symptoms } \\
\hline Headache & $13(61.9 \%)$ & & \\
\hline Nausea & $12(57.1 \%)$ & & \\
\hline Vomiting & $15(71.4 \%)$ & & \\
\hline Cranial nerve palsies & $9(42.9 \%)$ & & \\
\hline Ataxia & $12(57 \%)$ & & \\
\hline Somnolence & $7(33.3 \%)$ & & \\
\hline Syncope & $2(9.5 \%)$ & & \\
\hline Visual symptoms* & $5(23.8 \%)$ & & \\
\hline \multicolumn{4}{|l|}{ Neurosurgical intervention } \\
\hline EVD & 9 & & \\
\hline Ventriculoperitoneal shunt & 7 & & \\
\hline Tumor resection & 20 & & \\
\hline \multicolumn{4}{|l|}{$\begin{array}{l}\text { Time to short-term follow-up, } \\
\text { days }\end{array}$} \\
\hline Median & 12.5 & & \\
\hline Range & $1-232$ & & \\
\hline \multicolumn{4}{|l|}{ Time to long-term follow-up, yrs } \\
\hline Median & 3.76 & & \\
\hline Range & $1.80-6$ & & \\
\hline
\end{tabular}

formed at an outside facility as part of their initial evaluation. In 8 patients, intraoperative EVDs were placed immediately prior to tumor resection. In 5 patients, ICP was documented as high, but was not consistently recorded for all patients. Seven patients eventually required ventriculoperitoneal shunting, including 1 patient with a diffuse intrinsic pontine glioma.

The most common presenting symptoms included headache, nausea, vomiting, and ataxia (Table 1). All patients demonstrated at least some symptomatic improvement after removal of the obstructing mass and/or CSF diversion.

Four patients had formal ophthalmological examinations prior to resection. Five patients presented with preoperative visual symptoms, including blurry vision, vision loss, or diplopia. Of these 5 patients, 3 did not have any visual symptoms on follow-up examination. Postoperatively, 9 patients underwent ophthalmological evaluation with dilated fundoscopic examination and visual acuity testing. Three patients had persistent papilledema shortly after their primary procedure, one of whom continued to have chronic papilledema and sequelae of legal blindness almost 7 years after initial presentation. The other 2 patients demonstrated reduced visual acuity at short-term follow-up, which later showed near-complete resolution in both cases. Another patient had ongoing diplopia referable to trochlear and abducens palsies. Six patients had optic nerve atrophy on their most recent imaging, although only 2 were symptomatic.

\section{Imaging Findings}

Baseline DTI was performed prior to any surgical intervention in 19 patients. One patient had DTI performed only prior to resection of recurrent tumor. Short-term follow-up DTI was performed in 13 patients at a median 1 day (range 1-57 days) from neurosurgical intervention. Long-term follow-up DTI was performed in 13 patients at a median 1.8 years (range 3.03 months to 6 years) from the date of intervention.

\section{Ventricular Size}

The ventricles were visibly enlarged for all 21 patients and the bifrontal index ranged from 35.1 to $64.9 \mathrm{~mm}$ (median $41.9 \mathrm{~mm}$ ). Ventricular size was not correlated with patient age $(r=0.034, p=0.884)$. No significant correlation between ventricular size and preoperative optic nerve DTI parameters was found (MD: $\mathrm{r}=-0.161, \mathrm{p}=0.485$; FA: $r=0.235, p=0.305$ ).

The presence of high-intensity $\mathrm{T} 2$ signal within the optic chiasm was observed in 8 patients at initial presentation. No significant correlation was noted between high T2 signal and ventricular size $(r=-0.296, p=0.192)$ or optic nerve DTI metrics at initial presentation (MD: $\mathrm{r}=$ $-0.044, p=0.849$; FA: $r=0.022, p=0.925)$, or at shortterm (MD: $r=0.094, p=0.760$; FA: $r=0.131, p=0.669$ ) and long-term (MD: $\mathrm{r}=0.033, \mathrm{p}=0.915$; FA: $\mathrm{r}=-0.347$, $\mathrm{p}=0.245)$ follow-up.

\section{Diffusion Tensor Metrics}

Baseline and follow-up optic nerve DTI metrics in control and hydrocephalus cohorts are shown in Table 2. On baseline imaging, significant differences in optic nerve DTI metrics were observed between the hydrocephalus and control groups (Fig. 3). The hydrocephalus cohort demonstrated increased MD ( $\mathrm{p}<0.001)$, but decreased FA $(p=0.007)$ in bilateral optic nerve ROIs compared to controls: specifically, the median MD value of $1163 \times$ $10^{-6} \mathrm{~mm}^{2} / \mathrm{sec}$ (range 922-1589) among hydrocephalus patients was higher compared to $1022 \times 10^{-6} \mathrm{~mm}^{2} / \mathrm{sec}$ (range 869-1137) in controls. The hydrocephalus group had lower optic nerve FA of 0.50 (range 0.33-0.68) compared to controls with FA of 0.58 (range 0.49-0.69).

Figure 4 illustrates changes in optic nerve DTI metrics in the hydrocephalus patient cohort at initial presentation and short- and long-term follow-up. Following neurosur- 
TABLE 2. Baseline and follow-up optic nerve DTI in hydrocephalus and control cohorts

\begin{tabular}{|c|c|c|c|c|c|c|c|}
\hline \multirow[b]{2}{*}{ DTI Metric } & \multicolumn{2}{|c|}{$\mathrm{BL}(\mathrm{n}=21)$} & \multicolumn{2}{|c|}{ Hydrocephalus FU $(n=13)$} & \multicolumn{3}{|c|}{$p$ Value } \\
\hline & Control & Hydrocephalus & ST & LT & $\mathrm{BL}^{*}$ & ST vs BL† & LT vs BL† \\
\hline MD‡ & & & & & $<0.001$ & 0.006 & 0.011 \\
\hline Median & 1022 & 1163 & 1082 & 1021 & & & \\
\hline Range & 869-1137 & 922-1589 & $790-1268$ & 678-1569 & & & \\
\hline FA & & & & & 0.007 & 0.050 & 0.279 \\
\hline Median & 0.58 & 0.50 & 0.56 & 0.52 & & & \\
\hline Range & $0.49-0.69$ & $0.33-0.68$ & $0.34-0.75$ & $0.22-0.67$ & & & \\
\hline
\end{tabular}

$\mathrm{BL}$ = baseline; FU = follow-up; LT = long-term follow-up; ST = short-term follow-up.

* Comparison between baseline optic nerve DTI metrics of hydrocephalus and control cohort using the Mann-Whitney U-test.

$\dagger$ Wilcoxon signed-rank test for pairwise comparison made using baseline values of the hydrocephalus cohort.

$\ddagger$ Value $\times 10^{-6} \mathrm{~mm}^{2} / \mathrm{sec}$.

gical intervention, bilateral optic nerve MD significantly decreased by short-term follow-up $(\mathrm{p}=0.006)$ to a median value of $1082 \times 10^{-6} \mathrm{~mm}^{2} / \mathrm{sec}$ (range 790-1268). There was no significant difference between short- and long-term follow-up MD ( $\mathrm{p}=0.249)$. Optic nerve FA increased at short-term follow-up to 0.56 (range $0.34-0.75 ; p=0.050$ ) from initial presentation at 0.50 (range $0.33-0.68$ ). However, at long-term follow-up, FA decreased to 0.52 (range $0.22-0.67)$ with no significant difference compared to initial presentation $(p=0.279)$. Compared to age-matched controls, there were no differences in bilateral optic nerve MD or FA values at short- or long-term follow-up (MD: short-term $\mathrm{p}=0.287$, long-term $\mathrm{p}=0.511 ; \mathrm{FA}$ : short-term $\mathrm{p}=0.448$, long-term $\mathrm{p}=0.101$ ).

\section{Discussion}

In the present study, we demonstrate that DTI can quantitatively characterize underlying microstructural changes in the optic nerves of children presenting acutely with hydrocephalus. Furthermore, we show that these underlying alterations can be corrected with neurosurgical intervention, as depicted on short- and long-term followup imaging. To our knowledge, this is the first study to quantitatively identify DTI abnormalities of the optic nerves for this clinical population. Because DTI metrics can vary with age, we compared our hydrocephalus cohort to age-matched controls.

DTI is an advanced MRI technique that extracts the diffusivities of water parallel and perpendicular to the fiber tracts. In white matter, water diffusion occurs along the direction of the fibers, and is therefore anisotropic. Structural components, such as axons and myelin, influence anisotropy, and alterations in structural integrity are reflected in DTI metrics. ${ }^{2}$ MD directly correlates with cell loss and extracellular water extrusion, whereas FA reflects fiber integrity and is measured as the difference between the parallel diffusivity and the mean. ${ }^{2}$

Previous work in animal models demonstrated differential white matter vulnerabilities to obstructive hydrocephalus. ${ }^{4,13}$ In a feline kaolin-injection model of hydrocephalus, DTI metrics in various white matter structures, including the optic chiasm and tracts, were assessed before intervention as well as after early and delayed ven- tricular reservoir placement. ${ }^{4}$ Unlike in the corpus callosum, which demonstrated acute significant decreases in FA even with early reservoir placement, in the optic chiasm and tract FA was significantly lower only chronically with late reservoir placement. ${ }^{4}$ While MD increased in the acute setting for both reservoir groups, no alterations were found in the optic chiasm and tract between hydrocephalus animals and controls. ${ }^{4}$

Similar to previous studies, our results showed significant baseline alterations in DTI metrics in children with symptomatic hydrocephalus. However, prior studies largely focused on the corpus callosum, anterior and posterior limbs of the internal capsule, corticospinal tract, and superior and inferior longitudinal fasciculus. ${ }^{3,14}$ In one study of 19 patients with varying etiologies of hydrocephalus, the authors sought to identify whether DTI abnormalities could be correlated with symptom severity. ${ }^{2}$ Although they did not specifically evaluate the optic nerves, they did examine the optic radiations and found that all DTI metrics were significantly altered with hydrocephalus. ${ }^{2}$

In this study, we examined DTI metrics in the bilateral optic nerves both at baseline and after surgical interven-

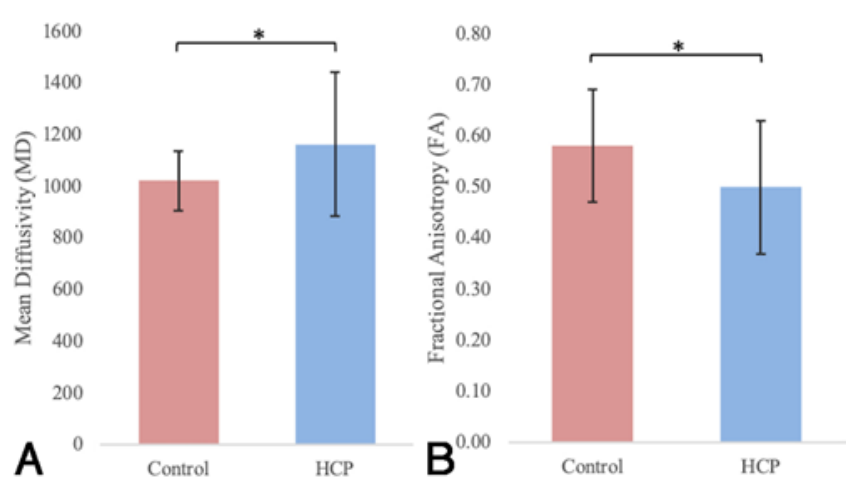

FIG. 3. Preoperative comparison of optic nerve DTI between the hydrocephalus (HCP) and control cohorts. MD in bilateral optic nerves is significantly higher in the hydrocephalus cohort $(\mathbf{A})$, while FA is significantly lower compared to healthy controls (B). * $p<0.05$. Error bars represent interquartile ranges around the depicted median value. 


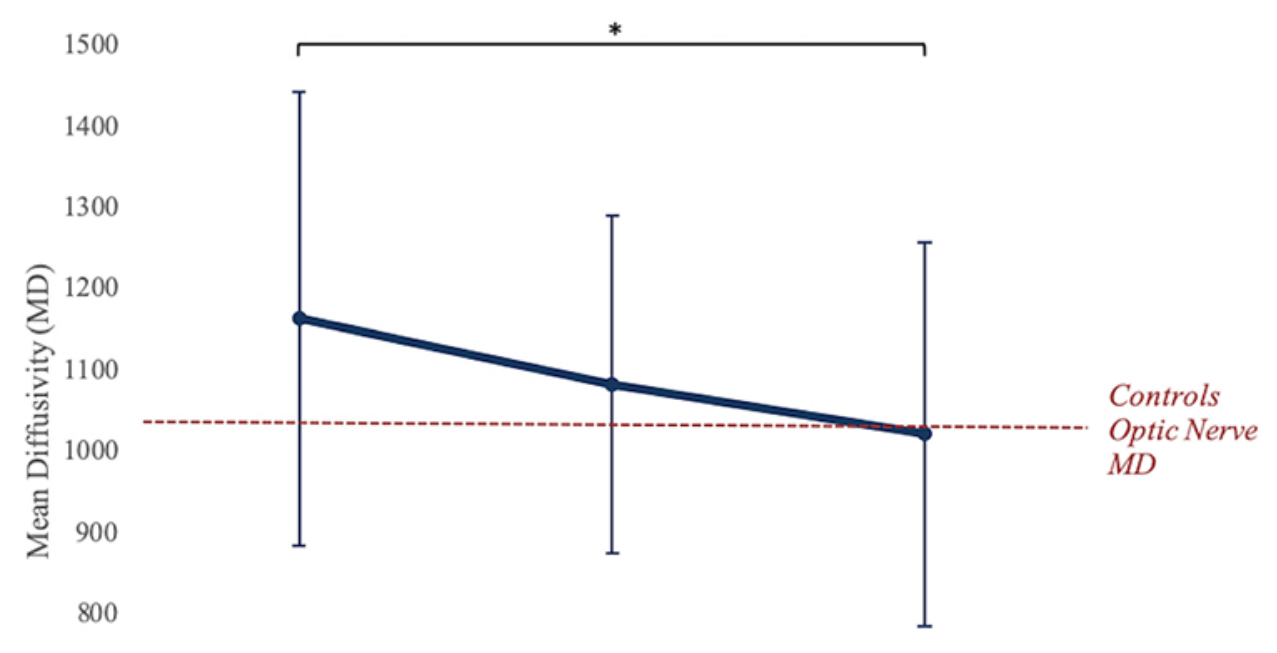

700

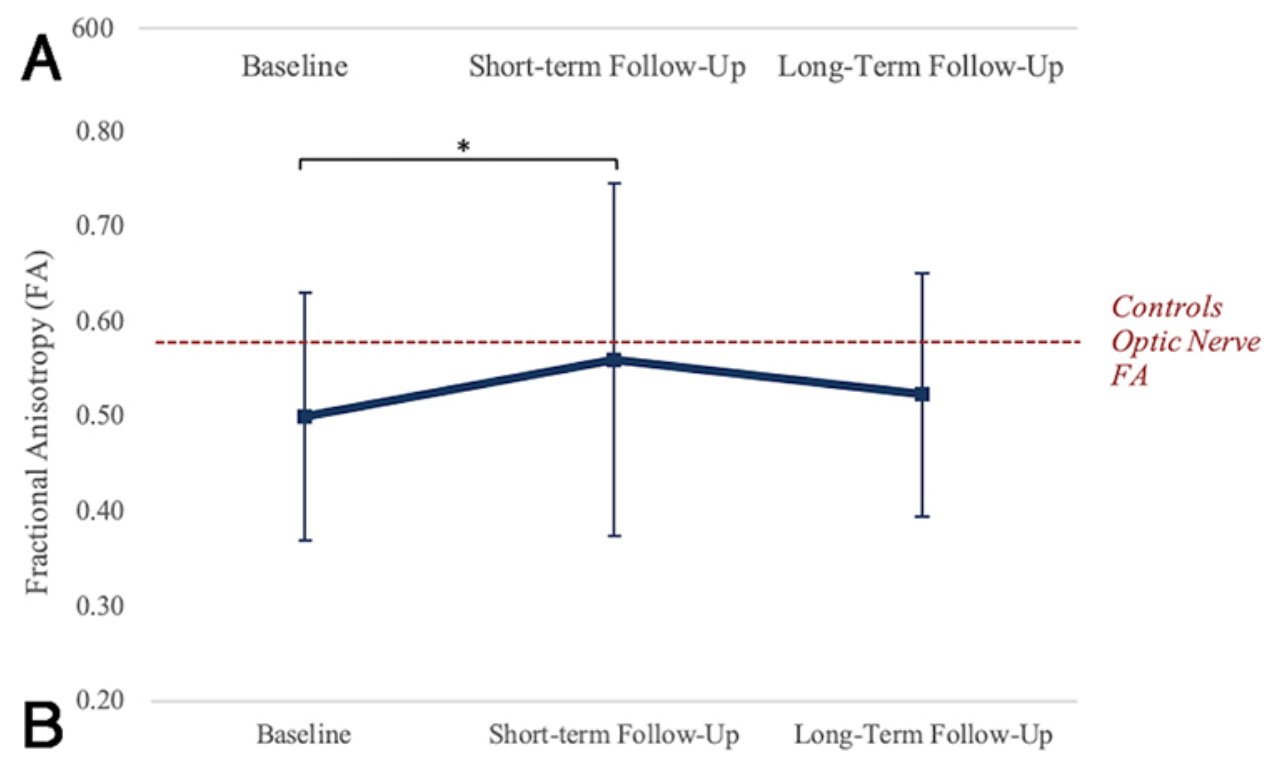

FIG. 4. DTI changes in hydrocephalus patients preoperatively and on short- and long-term follow-up. Dashed lines represent the median value of the control cohort. Error bars represent the interquartile range across the median value depicted as the center circular dot (for MD) or square (for FA). A: MD significantly decreased in bilateral optic nerves across follow-up time points. B: Optic nerve FA increased at both follow-up time points, reaching significance at short-term follow-up only. ${ }^{*} p<0.05$.

tion. Specifically, FA was reduced and MD increased in the bilateral optic nerves at the time of presentation with acute obstructive hydrocephalus. Interestingly, only 5 children reported visual symptoms, although these may have been underreported due to young age or changes in mental status. One patient who was blind on arrival was also overwhelmingly symptomatic and required emergency EVD placement the day prior to DTI and resection. This patient had persistent visual loss and optic nerve atrophy on follow-up imaging. These findings are concordant with previous literature showing frequent ophthalmological findings in children with uncompensated hydrocephalus. ${ }^{6}$ One study of 322 children with spina bifida and hydrocephalus found that $14 \%$ had papilledema, and $17 \%$ had optic nerve atrophy. Seventy percent of shunt malfunctions demonstrated ophthalmological evidence of increased ICP. ${ }^{6}$

Our results show near normalization of optic nerve DTI metrics both immediately after surgical intervention and on long-term follow-up. As shown in previous studies, restoration of diffusion tensor values to normal levels after either surgical decompression of an underlying obstructive lesion and/or CSF diversion suggests that some of these structural changes may be reversible. ${ }^{3}$ However, such "correctability" may be region- and etiology-specific. While one study demonstrated DTI improvements in the corona radiata after surgical intervention, no such changes were found in the optic radiations. ${ }^{2}$ Interestingly, the study also found greater correction of DTI metrics with ventriculoperitoneal shunting rather than ETV, which the study at- 
tributed to greater changes in ventricular size. ${ }^{2}$ Another study of 8 adults with idiopathic intracranial hypertension found a significant decrease in optic nerve FA after lumbar puncture, although baseline values were not compared to age-matched healthy controls. ${ }^{9}$ In our obstructive hydrocephalus cohort, optic nerve FA was reduced compared to controls, and increased closer to normal levels after surgical intervention.

Formal visual examinations, in particular fundoscopic examinations, can be challenging and unreliable in young children. In children with acute hydrocephalus, alterations in mental status and irritability compound these challenges. Numerous visual findings can occur in the setting of hydrocephalus, including abducens palsies, nystagmus, papilledema, optic nerve atrophy, and squinting. 6 More advanced imaging modalities such as optical coherence tomography (OCT) and confocal scanning laser ophthalmoscopy have also assisted in assessing papilledema and the degree of optic nerve damage. ${ }^{10,16}$ Our findings suggest that, even in the absence of overt visual complaints, the optic nerves show microstructural changes, and may therefore have potential clinical utility for optic nerve evaluation in pediatric hydrocephalus.

Our study has several limitations, including a small sample size. Also, only a small subset of patients underwent formal ophthalmological or funduscopic examinations, as this can be challenging in young children, particularly in those presenting with somnolence. Our findings suggest that even in the absence of overt visual symptoms, microstructural changes are indeed present in the optic nerves.

The small size of the optic nerve in general also renders DTI interrogation a technical challenge. We applied a GRAPPA-accelerated diffusion tensor method to reduce image distortion prevalent in regions of soft tissue-bone interface, such as the orbit. Additionally, we applied an inhouse retrospective motion-correction reconstruction algorithm to further reduce motion artifacts. ROI analysis of the optic nerve can be susceptible to reduced spatial resolution from partial volume averaging. These effects can be mitigated with reduced slice thickness and ROI extraction from multiple imaging planes. For this study purpose, a routine 3-mm slice thickness GRAPPA-DTI method was sufficient and ROI placement was optimized to ensure high SNR. As these scans were not isotropic or isovolumetric, multiplanar reformats were not performed as they would further reduce image quality and render evaluation of the small optic nerves less reliable. Higher-resolution diffusion MRI that combines a readout segmented-echo planar imaging technique could further reduce image distortion and may provide better orbital structural detail. ${ }^{19}$

Although we demonstrate group-level improvements in DTI metrics after surgical intervention, the role for DTI in other mechanisms of hydrocephalus, such as chronic shunted hydrocephalus, is unknown. Future studies could also combine optic nerve DTI with other imaging modalities such as OCT.

\section{Conclusions}

We contribute to the growing literature that DTI, and in particular, optic nerve DTI, may serve as a marker for papilledema in the setting of acute hydrocephalus and potentially identify microscopic tissue alterations associated with ongoing visual deficits. Future prospective and larger scale studies could further expand on our experience and examine the role of optic nerve DTI in the hydrocephalus workup and surveillance in neurosurgical practice.

\section{Acknowledgments}

We would like to acknowledge Michael Iv, MD, for his assistance with the imaging protocol and discussion of DTI applications. Kristen Yeom, MD, and Gerald Grant, MD, received the Stanford Bio-X Interdisciplinary Award to design and conduct the study.

\section{References}

1. Air EL, Yuan W, Holland SK, Jones BV, Bierbrauer K, Altaye $\mathrm{M}$, et al: Longitudinal comparison of pre- and postoperative diffusion tensor imaging parameters in young children with hydrocephalus. J Neurosurg Pediatr 5:385-391, 2010

2. Ben-Sira L, Goder N, Bassan H, Lifshits S, Assaf Y, Constantini S: Clinical benefits of diffusion tensor imaging in hydrocephalus. J Neurosurg Pediatr 16:195-202, 2015

3. Buckley RT, Yuan W, Mangano FT, Phillips JM, Powell S, McKinstry RC, et al: Longitudinal comparison of diffusion tensor imaging parameters and neuropsychological measures following endoscopic third ventriculostomy for hydrocephalus. J Neurosurg Pediatr 9:630-635, 2012

4. Eskandari R, Abdullah O, Mason C, Lloyd KE, Oeschle AN, McAllister JP II: Differential vulnerability of white matter structures to experimental infantile hydrocephalus detected by diffusion tensor imaging. Childs Nerv Syst 30:1651-1661, 2014

5. Forkert ND, Li MD, Lober RM, Yeom KW: Gray matter growth is accompanied by increasing blood flow and decreasing apparent diffusion coefficient during childhood. AJNR Am J Neuroradiol 37:1738-1744, 2016

6. Gaston H: Ophthalmic complications of spina bifida and hydrocephalus. Eye (Lond) 5:279-290, 1991

7. Hattingen E, Jurcoane A, Melber J, Blasel S, Zanella FE, Neumann-Haefelin T, et al: Diffusion tensor imaging in patients with adult chronic idiopathic hydrocephalus. Neurosurgery 66:917-924, 2010

8. Hayreh SS: Pathogenesis of optic disc edema in raised intracranial pressure. Prog Retin Eye Res 50:108-144, 2016

9. Hoffmann J, Kreutz KM, Csapó-Schmidt C, Becker N, Kunte $\mathrm{H}$, Fekonja LS, et al: The effect of CSF drain on the optic nerve in idiopathic intracranial hypertension. J Headache Pain 20:59, 2019

10. Hoye VJ III, Berrocal AM, Hedges TR III, Amaro-Quireza ML: Optical coherence tomography demonstrates subretinal macular edema from papilledema. Arch Ophthalmol 119:1287-1290, 2001

11. Hoza D, Vlasák A, Hořínek D, Sameš M, Alfieri A: DTIMRI biomarkers in the search for normal pressure hydrocephalus aetiology: a review. Neurosurg Rev 38:239-244, 2015

12. Keong NC, Pena A, Price SJ, Czosnyka M, Czosnyka Z, DeVito EE, et al: Diffusion tensor imaging profiles reveal specific neural tract distortion in normal pressure hydrocephalus. PLoS One 12:e0181624, 2017

13. Khan OH, Enno TL, Del Bigio MR: Brain damage in neonatal rats following kaolin induction of hydrocephalus. Exp Neurol 200:311-320, 2006

14. Patel SK, Vuong SM, Yuan W, Mangano FT: Anatomy and physiology-based magnetic resonance imaging in hydroceph- 
alus, in Limbrick DD Jr, Leonard JR (eds): Cerebrospinal Fluid Disorders: Lifelong Implications. Cham, Switzerland: Springer Nature, 2019, pp 131-152

15. Santoro JD, Forkert ND, Yang QZ, Pavitt S, MacEachern SJ, Moseley ME, et al: Brain diffusion abnormalities in children with tension-type and migraine-type headaches. AJNR Am J Neuroradiol 39:935-941, 2018

16. Schuman JS, Wollstein G, Farra T, Hertzmark E, Aydin A, Fujimoto JG, et al: Comparison of optic nerve head measurements obtained by optical coherence tomography and confocal scanning laser ophthalmoscopy. Am J Ophthalmol 135:504-512, 2003

17. Skare S, Newbould RD, Clayton DB, Albers GW, Nagle S, Bammer R: Clinical multishot DW-EPI through parallel imaging with considerations of susceptibility, motion, and noise. Magn Reson Med 57:881-890, 2007

18. Tuite GF, Chong WK, Evanson J, Narita A, Taylor D, Harkness WF, et al: The effectiveness of papilledema as an indicator of raised intracranial pressure in children with craniosynostosis. Neurosurgery 38:272-278, 1996

19. Yeom KW, Holdsworth SJ, Van AT, Iv M, Skare S, Lober RM, et al: Comparison of readout-segmented echo-planar imaging (EPI) and single-shot EPI in clinical application of diffusion-weighted imaging of the pediatric brain. AJR Am J Roentgenol 200:W437-W443, 2013

\section{Disclosures}

The authors report no conflict of interest concerning the materials or methods used in this study or the findings specified in this paper.

\section{Author Contributions}

Conception and design: Yeom, Shpanskaya, Grant. Acquisition of data: Yeom, Shpanskaya, Quon, Lober, Nair, Johnson. Analysis and interpretation of data: Yeom, Shpanskaya, Quon. Drafting the article: Yeom, Quon. Critically revising the article: Yeom, Shpanskaya, Quon, Lober, Cheshier, Edwards, Grant. Reviewed submitted version of manuscript: Yeom, Shpanskaya, Quon, Lober, Cheshier, Edwards, Grant. Approved the final version of the manuscript on behalf of all authors: Yeom. Statistical analysis: Shpanskaya. Study supervision: Yeom, Quon, Grant.

\section{Supplemental Information}

\section{Previous Presentations}

The contents of this manuscript were presented at the 2018 AANS Annual Scientific Meeting in New Orleans, Louisiana.

\section{Correspondence}

Kristen W. Yeom: Lucile Packard Children's Hospital, Stanford University School of Medicine, Stanford, CA. kyeom@stanford. edu. 